\title{
Preparing the COROT space mission: Building a photometric and variability database of stars in its field of view
}

\author{
P.J. Amado, R. Garrido
}

Instituto de Astrofísica de Andalucía-CSIC, Camino Bajo de Huétor, PO Box 3004, 18080 Granada, Spain

E. Poretti

INAF-Osservatorio Astronomico di Brera, Via E. Bianchi 46, 23807 Merate, Italy

E. Michel

Observatoire de Paris, LESIA, UMR 8109, place J. Jansen, 92195 Meudon, France

\section{Introduction}

The CNES/European space mission COROT will monitor asteroseismic targets located in selected fields to probe stellar interiors. Therefore, suitable candidate targets have to be searched for in order to optimize the scientific return of the mission. However, to be able to use the asteroseismic tools on the stars, their physical parameters must be known in advance. In this work, we detail the process of building a photometric database of all the stars brighter than $V=8.0$ in the field of view of COROT and the process of selecting suitable $\delta$ Sct and $\gamma$ Dor-type stars for the mission.

For an optimal selection of the seismology targets (for both COROT programs devoted to asteroseismology, i.e., the core and exploratory ones), it is essential to gather a priori as much information as possible on all potential candidates. With this aim, Strömgren-Crawford $u v b y-\mathrm{H} \beta$ and $\mathrm{Ca}$ II H\&K photometry were obtained for all of them. These data have been used to derive estimates of their effective temperatures, surface gravities and metallicities. These observations, together with high resolution echelle spectroscopy and high angular resolution imaging observations, are components of an ambitious ground-based program.

\section{The observing program}

We have used the 1.5-m and 0.9-m telescopes of the Sierra Nevada Observatory (OSN), managed by the IAA, to obtain $u v b y \beta$ and CaII H\&K photometry of all the stars with $V \leq 8.0$ among which will be, to a large extent, the primary and secondary COROT potential targets. At the $1.5-\mathrm{m}$ telescope, Ca II H\&K CCD photometry was gathered, while Strömgren and Crawford photoelectric photometry were obtained simultaneously in the $u v b y \beta$ filters at the $0.9-\mathrm{m}$ tele- 


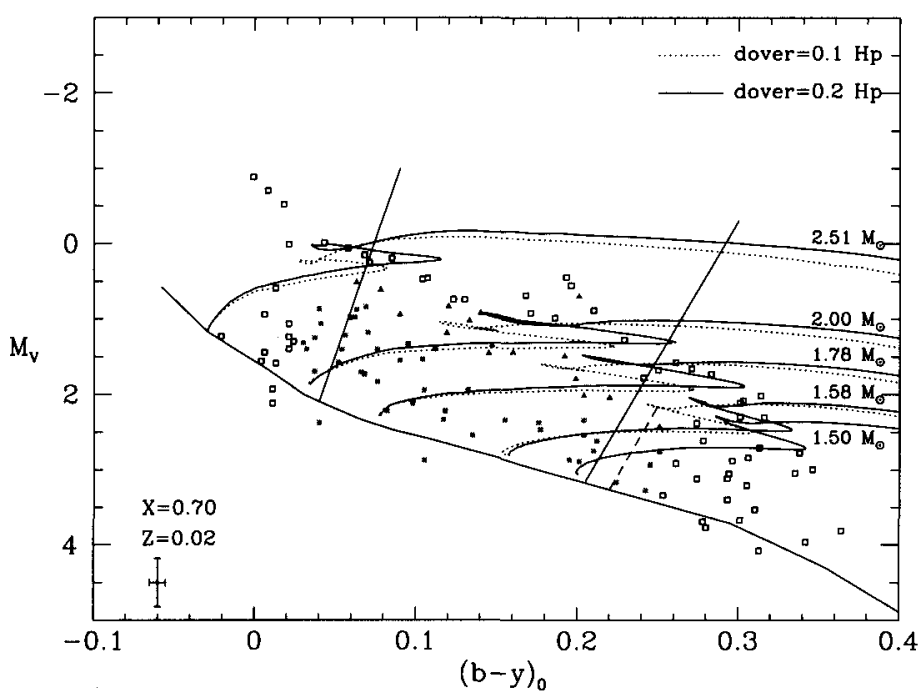

Figure 1. Hipparcos unreddened $M_{V}$ against dereddened $(b-y)_{0}$ colour indices for potential COROT targets in the Center direction. Dotted and solid lines indicate models with values of overshooting of $d_{\text {over }}=0.1$ and $d_{\text {over }}=0.2$, respectively. Solid squares represent stars that are certainly unevolved, independently of the value of the overshooting used. Solid triangles represent stars within the zigzags of the two sets of models and open squares are stars too evolved or outside of the instability strips. Mean photometric errors are given by the error bars in the lower-left corner of the plot.

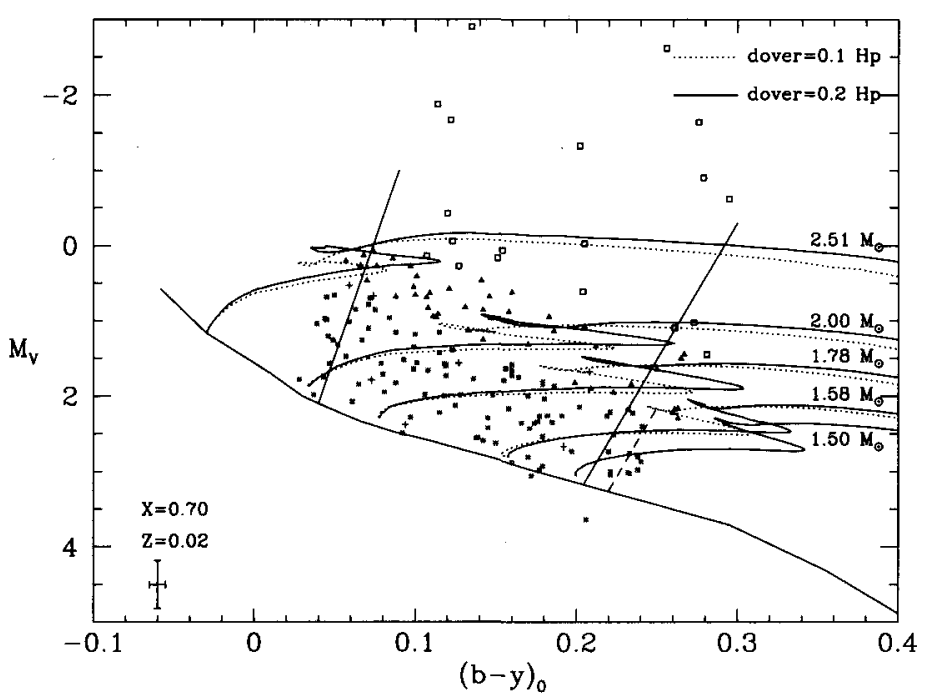

Figure 2. Same as in Fig. 1 but for the Anticenter direction. The crosses are stars with no Hipparcos parallaxes and therefore with $M_{V}$ determined from the photometry. 
HD 174966

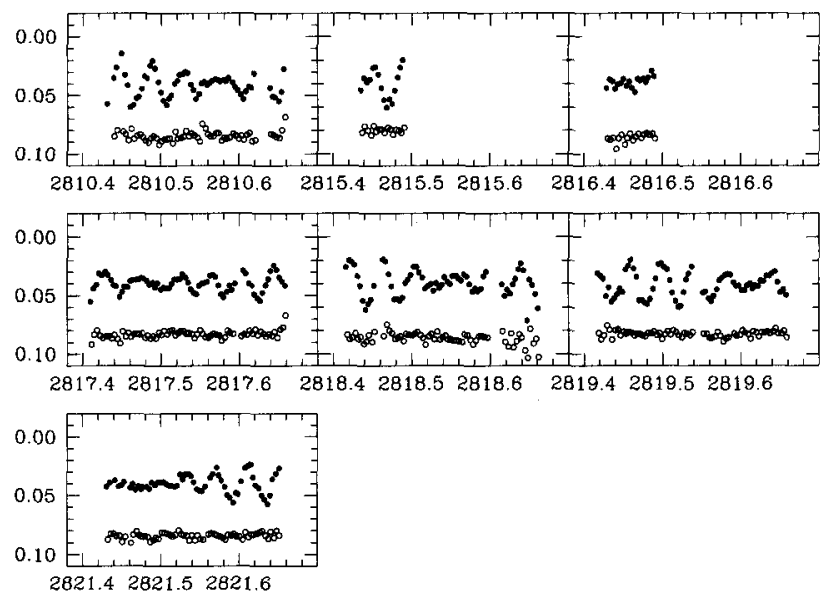

Figure 3. Strömgren $v$ differential (variable-comparison) magnitudes of the $\delta$ Sct star HD 174966 (solid circles) observed at OSN. Open circles are the differential light curves of the comparison stars HD 175272 and HD 175543. The standard deviation of the differential measurements between the comparison stars is 0.0038 mag.

scope. The whole dataset was reduced and calibrated to the respective standard systems by using specific software developed by our group (Amado et al., in preparation). The GAUDI data server provides access to the COROT Archive of the Ground-Based Seismology Program which has been developed by, and is maintained by the Laboratory for Space Astrophysics and Theoretical Physics (LAEFF), Spain (Solano et al. 2003). These data have been utilised to determine $T_{\text {eff }}, \log g$ and $[\mathrm{Fe} / \mathrm{H}]$ for all the possible targets of $\mathrm{B}, \mathrm{A}$ and $\mathrm{F}$ spectral types.

\section{Colour-Magnitude diagrams}

The observations mentioned above have, in part, led to the coverage of the Colour-Magnitude diagram (CMD) on and near the main sequence in the region of the instability strip. This has helped to enlarge the sample of known $\delta$ Sct and $\gamma$ Dor stars. In Figs 1 and 2, we present the diagrams for the stars within the instability strip in the field of view of COROT (Center and Anticenter directions). The main sequence is taken from Philip \& Egret (1980) and the $\delta$ Sct instability strip borders from Rodriguez \& Breger (2001). The red border of the $\gamma$ Dor instability strip is taken from Handler \& Shobbrook (2002); the blue border is well inside the $\delta$ Sct instability strip. We also added evolutionary tracks, considering five values for the mass in the range from 1.50 to $2.51 M_{\odot}$, and hydrogen and metal content of 0.70 and 0.02 , respectively. We calculated two sets of models for two different typical overshooting distances, i.e., $d_{\text {over }}=0.1$ and $d_{\text {over }}=0.2$ (see Claret 1995 for details). The limits of the instability strip 


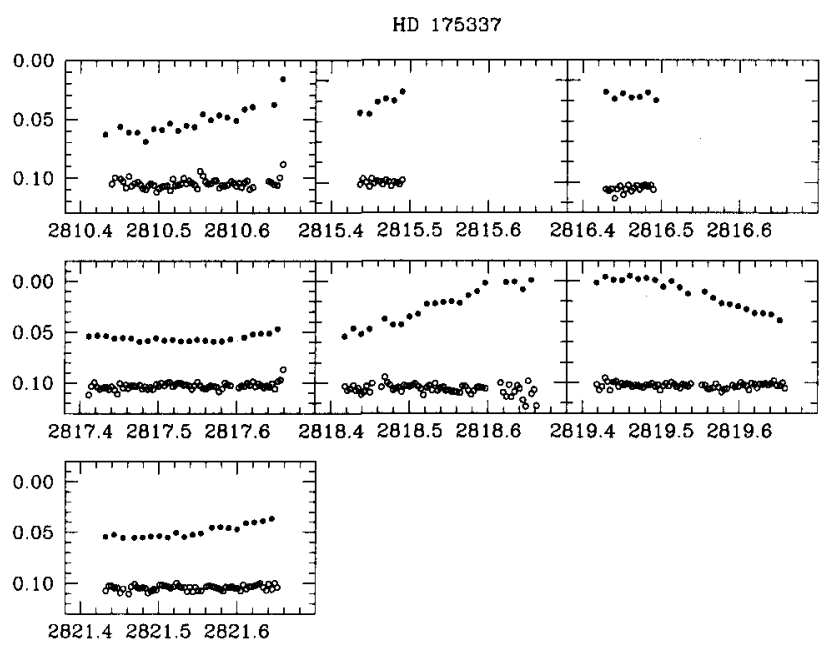

Figure 4. Same as Fig. 3 but for the $\gamma$ Dor star HD 175337

for stars to the left of the $\delta$ Sct instability strip blue edge and right of the $\gamma$ Dor instability strip red edge have been a little relaxed to take into account the errors in the photometric parameters.

\section{Enlarging the sample of known $\delta$ Sct and $\gamma$ Dor stars}

Once the potential targets to be monitored were selected from their position in the CMD, dedicated observing programs were carried out to try to make clear variability detections at the $0.005 \mathrm{mag}$ level. The sample of variable stars discovered in the Center direction is quite representative of the phenomenology we meet in the lower part of the instability strip. Fig. 3 shows an example of $\delta$ Sct variability: beat phenomena are clearly visible. Fig. 4 shows an example of the $\gamma$ Dor variability, much slower than the previous one. Both stars were observed during the two last weeks of 2003 June. The work is still in progress, since for some of the stars the variability has not yet been characterized in a precise way. For more examples of new $\delta$ Sct and $\gamma$ Dor stars discovered in the COROT ground-based observation program see Poretti et al. (2003).

\section{References}

Claret, A. 1995, A\&AS, 109, 441

Handler, G., Shobbrook, R.R. 2002, MNRAS, 333, 251

Philip, A.G.D., Egret, D. 1980, A\&A, 40, 199

Poretti, E., Garrido, R., Amado, P., et al. 2003, A\&A, 406, 203

Rodríguez, E., Breger, M. 2001, A\&A, 366, 178

Solano, E., González-Riestra, R., Catala, C., Baglin, A. 2002, in ESA SP-485: Stellar Structure and Habitable Planet Finding, p. 353 


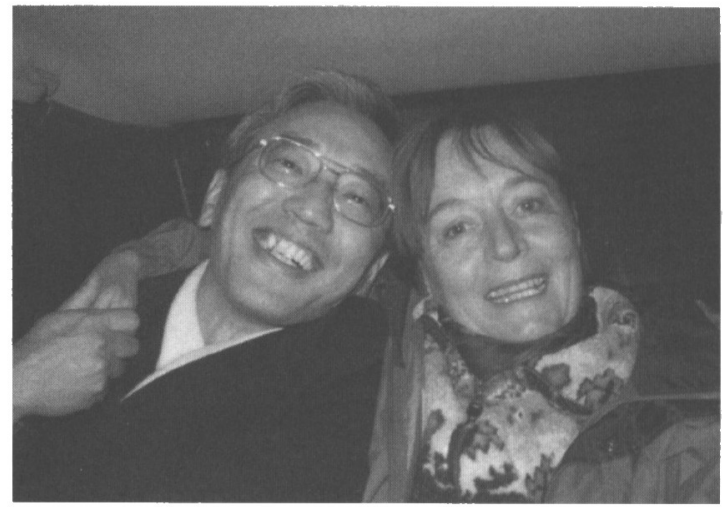

Hiromoto Shibahashi and Sylvie Vauclair

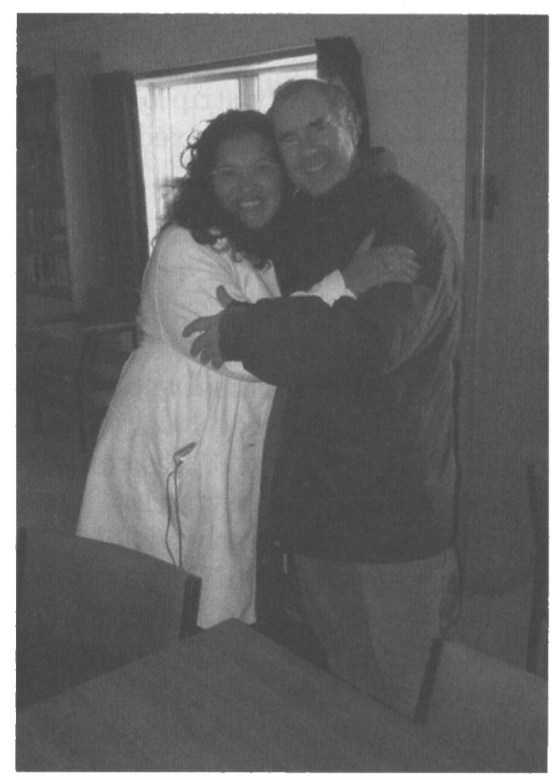

John Hearnshaw and Merieme Chadid 